\title{
Metabarcoding Reveals Diverse Endophytic Fungal Communities in Vaccinium Myrtillus Plant Organs and Suggests Systemic Distribution of Some Ericoid Mycorrhizal and DSE Fungi
}

Silvia Perotto ( $\square$ silvia.perotto@unito.it)

University of Turin

Stefania Daghino

Institute for Sustainable Plant Protection

Elena Martino

University of Turin

Samuele Voyron

University of Turin

\section{Research Article}

Keywords: Plant microbiome, endosphere, fungal community, Vaccinium myrtillus, Helotiales, endophytes, ericoid mycorrhizal fungi

Posted Date: January 25th, 2022

DOI: https://doi.org/10.21203/rs.3.rs-1257800/v1

License: (c) (1) This work is licensed under a Creative Commons Attribution 4.0 International License.

Read Full License 
1 Metabarcoding reveals diverse endophytic fungal communities in Vaccinium myrtillus plant organs and suggests systemic distribution of some ericoid mycorrhizal and DSE fungi

4 Stefania Daghino ${ }^{1}$, Elena Martino², Samuele Voyron ${ }^{1,2}$, Silvia Perotto ${ }^{2, *}$

$6{ }^{1}$ Institute for Sustainable Plant Protection, CNR, Strada delle Cacce 73, 10135 Torino and V. le 7 Mattioli 25, 10125 Torino, Italy.

$8 \quad{ }^{2}$ Department of Life Science and Systems Biology, University of Torino, V. le Mattioli 25, 10125, 9 Torino, Italy.

$11 *$ corresponding author

\section{Abstract}

13 Genome sequencing data revealed unexpected similarities among plant-interacting fungi belonging 14 to different ecological guilds. In particular, the sequenced genomes of ericoid mycorrhizal fungi 15 (ErMF) showed closer similarities with genomes of plant endophytes than with those of other mycorrhizal fungi. ErMF are typically associated with roots of plants in the Ericaceae, but it has never been investigated whether they also colonize other organs of their natural hosts. Here, we applied a metabarcoding approach to describe the fungal community associated with the different organs of Vaccinium myrtillus plants collected in the field. Taxa in the Helotiales and Sebacinales, known to include ErMF, characterize the root endosphere, together with Agaricales and Lecanoromycetes, while the stems were enriched in Agaricomycetes, Tremellomycetes and Pleosporales, the leaves were enriched in Sordariomycetes, Hysteriales, and the flowers were enriched in Dothideomycetes. Operational Taxonomic Units attributed to known or putative ErMF and Dark Septate Endophytic fungi, namely Pezoloma ericae, Meliniomyces spp. and Phialocephala fortinii, were found in all the plant organs. The ErMF Oidiodendron sp. was rarely detected in organs other than roots in field samples, but we could detect its presence in the aboveground organs of $V$. myrtillus grown in vitro. This first report of ErMF colonizing the above-ground tissues of the host plant mirrors their evolutionary closeness with endophytes and increases the list of fungi found to occupy several niches.

\section{Keywords:}

33 Plant microbiome, endosphere, fungal community, Vaccinium myrtillus, Helotiales, endophytes, 34 ericoid mycorrhizal fungi. 


\section{Introduction}

Plants live closely associated with complex microbial communities, or microbiota, that colonize the plant surfaces (e.g., rhizosphere and phyllosphere) as well as internal tissues (the endosphere), and include nematodes, fungi, unicellular eukaryotes, bacteria, archaea and viruses ${ }^{1}$. The plantassociated microbiota can play a key role for plant health, productivity and development, and a plant with its associated microbiota, the "holobiont", can be considered as a single entity that evolves in the environment and time, thanks to the co-evolution of the single components interacting with each other ${ }^{2}$.

Microorganisms inhabiting the plant internal tissues for at least part of their lifetime are termed endophytes ${ }^{1}$. In particular, endophytic fungi are functionally dominant in the plant microbiota; they are ubiquitous and have been found in all species of plants studied to date ${ }^{3}$. Endophytic fungi live in the host tissues without causing evident symptoms ${ }^{4}$ and are often considered to be beneficial to their host plants because they may provide resistance against pathogens and insect herbivory ${ }^{5}$. They can also confer stress tolerance, such as salt and heat tolerance ${ }^{6}$ and promote plant root formation and shoot growth ${ }^{7}$. On the other hand, endophytic fungi could become pathogens under stressful conditions, or they could have long latent periods ${ }^{8}$.

Rodriguez et al. ${ }^{3}$ classified endophytic fungi according to their colonization pattern and phylogeny. Class I endophytes, also known as clavicipitaceous endophytes, includes phylogenetically related species that form systemic intercellular infections in the shoots of some grasses, being primarily vertically transmitted. Class II, III and IV include a taxonomically and functionally highly diverse group of non-clavicipitaceous species. Class II species can extensively colonize both above- and below-ground plant tissues, can be transmitted both horizontally and vertically via seed coats, seeds or rhizomes and confer habitat-specific stress tolerance to host plants ${ }^{6}$. Class III species are characterized by a high diversity within a single plant, where they are limited to above-ground tissues with highly localized infections, and can be horizontally transmitted. Class IV species, namely the Dark Septate Endophytic (DSE) fungi, are restricted to the roots, which they colonize extensively in a wide range of host plants, and are horizontally transmitted. This classification of fungal endophytes excluded the mycorrhizal fungi because, in addition to internal root tissues, these fungi grow outside the rhizosphere into the soil. Mycorrhizal fungi colonize the plant root tissues, where they form intimate symbioses whose morphological and functional features depend on the plant and the fungal taxonomic position ${ }^{9}$. The formation of specialized fungal structures within the plant tissues also excluded mycorrhizal fungi from the definition of endophytes given by Wilson ${ }^{10}$ "fungi or bacteria which, for all or part of their life cycle, invade the tissues of living plants and cause unapparent and asymptomatic infections entirely within plant tissues but cause no symptoms 
of disease”, whereas they were included in the definition of endophytes proposed by Hardoim and colleagues $^{1}$, thus indicating the complexity of defining boundaries between different fungal guilds. Irrespective of the definition and spectrum, many endophytic fungi seem to be unequally distributed in the different plant compartments. A similar pattern has been found in the diversity and distribution of bacteria associated with plant surfaces and internal plant tissues, both above- and below-ground, where it has been suggested that these plant compartments may represent a major selective force that shapes the composition of plant-associated microbiota ${ }^{2}$. When compared to communities of bacterial endophytes, that have been widely investigated, variation in the fungal communities within the different plant niches is still poorly known, and although several studies have focused on the plant-soil interface, less is known about the patterns of fungal diversity in the different plant compartments ${ }^{11}$. It is for example unclear whether the distribution in the plant depends on the taxonomic position of the fungus, or on specific constraints posed by the different plant compartments. To increase our knowledge on the fungal communities associated with aboveand below-ground plant tissues, and to address specific questions on the distribution of some key components of plant-associated fungi, we have investigated the diversity of the fungal community colonizing the internal tissues of different organs of Vaccinium myrtillus (Ericaceae) plants, a species known to form a specific mycorrhizal symbiosis.

Plants belonging to the Ericaceae family, encompassing 4426 species and around 129 genera ${ }^{12}$, represent important components of the heathland flora and some open forest communities worldwide. These geographically and climatically disparate habitats rely on soils that are usually very poor in mineral nutrients but can be enriched in aromatic compounds and potentially toxic metals, made readily available by the generally low $\mathrm{pH}^{13}$. The adaptation of Ericaceae to these stressful habitats has been largely attributed to the ability of their associated mycorrhizal fungi to increase the host plant fitness ${ }^{14}$. The role of non-mycorrhizal fungal endophytes in the adaptation of Ericaceae to stressful conditions is far less understood, although Class IV endophytes, namely the DSE fungi ${ }^{3}$, are commonly isolated from the roots of ericaceous plants ${ }^{15-19}$ and inoculation with DSE fungi under controlled conditions enhanced plant performance ${ }^{20}$. Furthermore, some DSE fungi seem to have a potential to form ErM $^{21}$.

Besides playing a crucial ecological role in heathland habitats, some genera of Ericaceae have a commercial interest as agronomic cultures in the flower and horticultural industry, both as food and nutraceutical sources, thanks to their richness in secondary metabolites ${ }^{22}$. Ericoid mycorrhizal fungi have been demonstrated to influence not only plant fitness in the field, but also some plant phenotypic traits, such as flower size and fruit number and quality ${ }^{23}$. Fungal endophytes from ericaceous plants may also be potential new sources of antimicrobial compounds. For example, 
103 Tong and co-workers ${ }^{24}$ studied the inhibitory effects against pathogenic bacteria of fungal

104 endophytes isolated from $V$. dunalianum var. urophyllum, a medicinal blueberry used in southern 105 China.

106 The fungal communities associated with ericaceous plants have been mainly investigated in the 107 roots by culture-dependent methods, most studies being focused on the isolation and identification 108 of ErMF. Ericoid mycorrhizal fungi form typical symbiotic hyphal coils within the root epidermal 109 cell and are mainly ascomycetes in the class Leotiomycetes. Pezoloma ericae (formerly 110 Rhizoscyphus ericae, Hymenoscyphus ericae and Pezizella ericae ${ }^{25}$ was the first species 111 experimentally confirmed as $\mathrm{ErMF}^{26}$. Later, many sterile isolates from Ericaceae roots were 112 classified in a single species complex known as “ $R$. ericae aggregate” ( REA $^{27}$ ) by molecular 113 methods. The REA includes confirmed ErMF species such as Meliniomyces variabilis and $M$. 114 bicolor, as well as ectomycorrhizal species, such as Cadophora finlandia, and other mycorrhizal 115 and non-mycorrhizal endophytes ${ }^{28}$. More recently, a taxonomic revision of the REA has been 116 proposed, reducing Meliniomyces spp. and Pezoloma ericae to synonymy under the Hyaloscypha 117 genus $^{29}$. Outside the REA, fungi belonging to the species Oidiodendron maius have been often 118 isolated from mycorrhizal ericaceous plants and shown to form typical hyphal coils ${ }^{30}$.

119 Other ascomycetes have been sporadically reported to form hyphal coils in the roots of ericaceous 120 plants in vitro and are considered as putative ErMF, although the mycorrhizal function of some of 121 these associations is still under debate (see ${ }^{28}$ and references therein). They include: some non-REA 122 Helotiales that can form functional ErM, isolates in the genus Leohumicola, Acremonium strictum, 123 Geomyces pannorum, some DSE fungi of the Phialocephala-Acephala applanata complex (PAC ${ }^{21}$ ), 124 isolates with affinities to the genera Capronia, Cadophora, Cryptosporiopsis and Lachnum, fungi 125 belonging to an unnamed lineage in the Chaetothyriomycetidae ${ }^{31}$.

126 Basidiomycetes species in the genus Serendipita (Sebacinales, Agaricomycetes) are also common 127 inhabitants of ericaceous roots, where they form typical hyphal coils ${ }^{32}$. A species in the order 128 Trechisporales (Agaricomycetes), identified by Vohník and colleagues ${ }^{33}$ from Vaccinium spp., has 129 been considered as a putative ErMF because it forms intracellular structures with a unique 130 morphology described as a "sheathed-ericoid” mycorrhiza.

132 Whereas the root-associated fungal communities of ericaceous plants have been investigated by 133 culture-dependent and independent methods in many recent studies ${ }^{16,17,19,34-38}$, few investigations 134 have focused on the fungal diversity in the above-ground organs. $\mathrm{Li}$ and colleagues ${ }^{39}$ analyzed the 135 diversity of the endophytic fungal community from fruits, leaves and branches of $V$. dunalianum 136 var. urophyllum (known as South China blueberry), whereas Koudelkova and colleagues ${ }^{40}$ isolated 
137 fungal endophytes from Rhododendron tomentosum leaves. Thus, information about the fungal 138 diversity characterizing plant compartments different from the roots is very limited in the Ericaceae. Here, we investigated by metabarcoding the endophytic fungal diversity of both below- and aboveground organs of field collected plants of V. myrtillus (European blueberry), with the aim to verify if different plant compartments (i.e., roots, stems, leaves and flowers) harbor similar or significantly different communities of endophytic fungi.

In addition, we addressed specific questions on the distribution of ErMF and DSE fungi in the host plant. Ericoid mycorrhizal fungi have been sometime observed as endophytes in non-ericaceous hosts $^{41}$ and recent data indicate that some genomic features of sequenced ErMF ${ }^{42}$ and the root DSE fungus Phialocephala subalpina ${ }^{43}$ are similar to those of other endophytes, with an expansion of the repertoire of Carbohydrate Active enZymes (CAZymes) and an unusually high number of genes coding for polyketide synthases involved in the biosynthesis of bio-active secondary metabolites ${ }^{42}$. Thus, we hypothesize that some root-associated fungi may be more versatile in their trophic strategies and colonization potential than traditionally thought. A further aim of this work was therefore to verify if fungi typically described as being restricted to the root endosphere of ericaceous plants, like ErMF and DSE fungi, can also colonize the above-ground plant organs.

\section{Results}

Fungal diversity associated with the different plant organs

The fungal communities associated with the four different organs of $V$. myrtillus were revealed by high-throughput sequencing of the fungal ITS2 region. After removal of low-quality reads, we obtained in total 2,863,742 high quality reads (maximum counts per sample: 188,914; minimum counts per sample: 93,654) corresponding to 1,621 Operational Taxonomic Units (OTUs; 97\% similarity), among which 1,186 had $\geq 2$ counts. After discarding OTUs with low counts (less than 10 reads) and low standard deviation (see material and methods), 749 OTUs were retained.

The alpha diversity of fungal communities in the four different plant organs analyzed (i.e., roots, stems, leaves and flowers) was assessed by calculating the Chao1 and Shannon indices. The Chao1 index, which estimates richness based on taxa abundance, showed no significant differences among organs (Kruskal-Wallis p-val=0.08; Supplementary Fig. S1), while the Shannon index, that considers both richness and evenness (abundance distribution across species), revealed a significant difference among organs (Kruskal-Wallis p-val=0.047; Supplementary Fig. S1), with the highest fungal diversity in leaves. No significant differences have been found in the alpha-diversity values of the different samples of each organ. 
170 Beta-diversity was estimated by NMDS based on Bray-Curtis dissimilarities and showed that the

171 fungal communities of stems, leaves and flowers were partially overlapping, whereas the fungal 172 community from the root samples clustered separately in the ordination space (Permanova F173 val=4.349, $\mathrm{R}^{2}=0.449$, p-val <0.001, NMDS stress=0.134; Fig. 1).

174 At the phylum level (Supplementary Fig. S2), the fungal population associated with V. myrtillus 175 plants was dominated by Ascomycota (overall 50\% of the total reads), followed by Basidiomycota 176 (overall 15\%) and by all the other phyla with percentages below 1\% (Glomeromycota, 177 Mortierellomycota, Mucoromycota, Olpidiomycota). A large percentage of the total reads (overall 178 32\%) corresponded to unidentified and not assigned phyla. The phylum Basidiomycota was significantly more abundant in stems than in flowers and leaves (Supplementary Fig. S2). At the class level (Fig. 2a), Dothideomycetes were the most abundant (overall 29\%), followed by Leotiomycetes (14\%), Agaricomycetes (11\%) and by the other classes with percentages below the $2,1 \%$. Overall, $35 \%$ of the total reads corresponded to unidentified and not assigned classes. The classes Leotiomycetes, Dothideomycetes, Tremellomycetes and Agaricomycetes showed significant differences in their abundance across the different organs, as shown in Fig. 2b. In particular, the class Leotiomycetes was significantly more abundant in roots than in all the other organs. At the genus level (Supplementary Fig. S3), 76\% of taxa in all organs were unidentified or not assigned, whereas the most abundant identified genus was Athelia (overall 5\%) followed by Phialocephala (overall 3.5\%) and Cladosporium (overall 1.8\%). The abundance of the Athelia genus was significantly higher in stems (Supplementary Fig. S3). Among the genera including known ErMF, we found Pezoloma (0.7\%), only represented by P. ericae (Supplementary Table S1), Meliniomyces (0.7\%), represented by the ErMF $M$. bicolor and $M$. variabilis and by the non-ErMF $M$. vraolstadiae, and Oidiodendron (0.01\%) represented by the ErMF O. maius and by the non-ErMF O. griseum. In addition, among those that are considered putative ErMF according to the literature ${ }^{28}$, we found Lachnum (0.25\%), Capronia (0.03) and Cryptosporiopsis (0.01\%). number of significantly different taxa were found when roots were compared with all the other organs. In particular, the orders Helotiales and Leucosporidiales were always more abundant in roots than in the other organs, while Dothideales and Capnodiales, both in the class Dothideomycetes, were less abundant in roots. Sebacinales were more abundant in roots than in leaves and stems. Atheliales were more abundant in stems than in the other organs, while Polyporales were more abundant in leaves and Capnodiales were more abundant in flowers. The LefSe score (Linear discriminant analysis Effect Size ${ }^{44}$ ) was used to estimate differences in the relative taxa abundance among organs at the class, order and genus level (Fig. 4). Few taxa were 
204 identified that could be considered as markers of the different organs. Roots were enriched

205 (LogLDA>3) in the classes Leotiomycetes (with the order Helotiales and the genera Phialocephala 206 and Meliniomyces) and Lecanoromycetes, in the orders Sebacinales (with the genus Serendipita) 207 and Agaricales. Stems were enriched in the classes Agaricomycetes (with the order Atheliales and 208 the genus Athelia) and Tremellomycetes, and in the order Pleosporales. Leaves were enriched in the 209 class Sordariomycetes and in the order Hysteriales, while flowers were enriched in the class 210 Dothideomycetes (with the order Capnodiales and the genus Cladosporium; Fig. 4a-b-c). Principal 211 Component Analysis (PCA) of OTUs distribution (Fig. 4d) showed that the differences between the 212 organs were driven by few single OTUs. In particular, roots were characterized by OTUs 39, 716, 213 719, 736 (all unidentified, with the exception of OTU716 assigned to Phialocephala fortinii), 214 flowers by OTUs 98, 591, 621 (the first being unassigned, while the other two being assigned to 215 Cladosporiaceae), stems by OTUs 457, 2270, 704, 411 (the first being assigned to Athelia, the 216 second and third to Melanommataceae in the Dothideomycetes, while the last being unassigned).

217 A correlation network analysis based on Pearson's statistics, which determines whether linear 218 relationships exist between two taxa, showed in the roots a significant co-occurrence of the classes 219 Leotiomycetes, Eurotiomycetes and Geoglossomycetes (Supplementary Fig. S4). The same analysis 220 at the genus level in the roots showed co-occurrence of genera including known ErMF species, 221 namely within Oidiodendron, Meliniomyces and Serendipita (Supplementary Fig. S4). In addition, 222 such genera including ErMF species showed a significant co-occurrence with Basidiomycetes 223 known to be ectomycorrhizal on tree species, such as Suillus, Russula, and Lactarius, and with the 224 DSE Phialocephala.

225 We found 214 core OTUs present in the four organs (Supplementary Fig. S5). Among them, the 226 most abundant genera were Phialocephala, mainly detected in roots, Athelia, mainly detected in 227 stems, and Cladosporium, mainly detected in flowers. Among the core OTUs were also few genera 228 including known and putative ErMF species, such as Pezoloma (with the single species P. ericae), 229 Meliniomyces (with the two ErMF species M. bicolor and M. variabilis), Geomyces, members of the 230 PAC (Phialocephala, Cadophora) as well as some ectomycorrhizal fungi (Supplementary Fig. S5). 231 The double-clustering analysis of the core OTUs showed that root and stem samples form distinct 232 clusters, suggesting that the abundance and distribution of the core OTUs changes in these organs, 233 while (not surprisingly) is more similar in flowers and leaves (Fig. 5).

Fungal isolation from V. myrtillus roots and phylogenetic analysis

Subsamples of roots, one from each sample 1, 3 and 4, were used for the isolation of cultivable fungi. In total, 44 different morphotypes were obtained in axenic culture and 37 of them could be 
taxonomically assigned by molecular analysis (Supplementary Table S2). The identity of 15 isolates assigned to the Helotiales (Supplementary Table S2) was further confirmed by both morphological observations and by phylogenetic analysis of the ITS2 sequences (Supplementary Fig. S6-S7). They were assigned to the species Oidiodendron maius, O. chlamydosporicum, O. tenuissimum, O. griseum and Cadophora luteo-olivacea. This mirrors the finding of some OTUs from V. myrtillus roots that were attributed to the same fungal species by phylogenetic analyses (Supplementary Fig S6-S7). OTUs attributed to the REA (Supplementary Fig. S7) have been detected mostly in roots, but they were also found in other plant organs.

Plant colonization by Helotiales isolates in vitro.

The 15 Helotiales isolates were tested for their ability to colonize V. myrtillus plants in vitro. The three O. maius strains formed typical coils in the root epidermal cells (Fig. 6a-b-c), whereas the two C. luteo-olivacea colonized the root tissues but did not form specific fungal structures (Fig. 6e-f). The other Oidiodendron species associated with the roots but did not form typical mycorrhizal coils (Fig. 6d).

We checked for the presence of three of the fungal isolates in the above-ground organs of plants grown in axenic conditions. We extracted total DNA from pooled stems and leaves, using the same protocol described for the field-collected plants and tested it by PCR with primers designed on the fungal ITS2 region (Supplementary Fig. S8). PCR amplification of total DNA extracted from plants inoculated with C. luteo-olivacea yielded a single amplicon corresponding to Cadophora sp. By contrast, PCR amplification of total DNA extracted from plants inoculated with the O. maius and $O$. tenuissimum isolates produced two amplicons that were attributed by Sanger sequencing to Vaccinium sp. and Oidiodendron spp., respectively (Supplementary Table S3).

\section{Discussion}

The plant internal tissues represent a unique ecological niche where some distinctive fungal endophytic species may live. The endophytic association plays an important role in the adaptation to the environment of both plants and fungi, together with the other organisms that constitute the holobiont. It has been suggested that plants select their microbiome for traits rather than taxonomy, because it provides many functions that are a part of an 'accessory genome' and that may be distributed across many different taxa ${ }^{2}$.

Here, we have used a culture-independent approach to investigate the endophytic fungal communities associated with different organs of V. myrtillus (Ericaceae) plants collected in an alpine habitat. 
272 At a coarse taxonomic level, the fungal population was dominated by Ascomycetes, followed by

273 Basidiomycetes. This is similar to the results of previous studies on the root-associated fungi of

274 Ericaceae ${ }^{16,17,19,34-36}$ but in contrast with the report of Trivedi and colleagues ${ }^{2}$ that, based on the

275 analysis of metabarcoding datasets from different angiosperms, stated that the endospheric fungal

276 community was dominated by Basidiomycetes.

277 Association of some fungal endophytes with specific host tissues has been observed in some plant

278 species $^{45}$. Similarly in V. myrtillus, we showed that the different organs shape the endophytic fungal

279 community. The analysis of beta-diversity revealed that the fungal community colonizing the root

280 endosphere was particularly different from the others, possibly because of the closeness and

281 influence of the rhizopheric soil.

282 Alpha-diversity indices suggest a similar degree of fungal diversity within the V. myrtillus organs,

283 except for the diversity associated with leaves, that was higher when evenness was taken into

284 consideration by the Shannon index, in line with previous reports ${ }^{2,39}$.

285 Relative abundance of lower rank taxa revealed that the Helotiales were more abundant in roots

286 than in the other organs, and Phialocephala and Meliniomyces genera could be considered as

287 biomarkers of the root compartment. In particular, one of the OTUs that determined the divergence

288 of the root compartments from the other plant compartments was assigned to Phialocephala

289 fortinii. This species belongs to the group of the DSE fungi and forms with A. applanata the so-

290 called P. fortinii s.l. - A. applanata species complex (PAC), often found to be associated with

291 Ericaceae roots ${ }^{15-19}$. Meliniomyces comprises species known as ErMF, such as M. bicolor and $M$.

292 variabilis $^{28}$, both found in our dataset. Sebacinales were also more abundant in roots than in leaves

293 and stems, with Serendipita as biomarker of the root compartment. Sebacinales have been already

294 reported as common fungi in Vaccinium spp. roots ${ }^{15,19,34}$ and encompass ubiquitously distributed

295 taxa found as symbionts in diverse mycorrhizal types, ranging from ectomycorrhiza to ericoid and

296 orchid mycorrhiza, and as root endophytes. Species belonging to the Leucosporidiales, found to be

297 more abundant in roots that in the other organs, have been already observed as leaves and stems

298 endophytes both in grasses and in woody plants ${ }^{46,47}$ but, to our knowledge, they have never been

299 reported from roots.

300 Among the dominant genera in the roots, we detected fungal endophytes belonging to the

301 Neonectria genus, as already reported by Zhang and coworkers ${ }^{18}$ in blueberry roots. We also

302 identified, in the root compartment, two typically ectomycorrhizal fungal genera, Russula and

303 Hygrocibe. Ectomycorrhizal fungal genera (Russula, Tomentella, Rhizopogon, Thelephora,

304 Cenococcum) were also previously found in the roots of V. carlesii by Zhang and colleagues ${ }^{34}$. 
305

All the analyses suggested the dominance of fungi in the order Atheliales in the stems, with the Athelia genus as stem biomarker. This genus corresponded to a single OTU, very abundant in the stems of all samples. Strains belonging to the Athelia genus have been reported as psychrophilic fungi associated with cold-stored food ${ }^{48}$ and subalpine fir foliage ${ }^{49}$, and as destructive lichenicolous basidiomycete $^{50}$. Athelia was previously reported in the rhizophere of Vaccinium angustifolium ${ }^{38}$, and here we found it in the root tissues as well, although represented by few reads. The presence of this single OTU in the above-ground organs of all samples suggests that it might be a fungus associated with the understorey vegetation of the alpine field analyzed. The same hypothesis may be proposed for 16 OTUs, mainly attributed to Ascomycota and detected in all samples, but a more extensive analysis on different plant species from the same field site would be necessary to support it. Capnodiales were relatively more abundant in the flowers and the Cladosporium genus could be considered as a biomarker of this compartment. Cladosporium species have been reported from $V$. dunalianum fruits and branches ${ }^{39}$, as well as from blossom blight in strawberry ${ }^{51}$. The flowers used in our study did not show symptoms of pathogen infections.

The plant core microbiota consists of those members of the microbial community that are ubiquitous in the plant compartments. Few dominating taxa in a single V. myrtillus organ turned out to be present, although with lower reads numbers, in the other organs as well, being part of the host core microbiota. This was the case, for example, for the Phialocephala, Athelia and Cladosporium genera. The identification of Phialocephala, represented by the single assigned species $P$. fortinii, in the core microbiota was interesting, as this DSE fungus is generally reported as a root-specific endophyte ${ }^{52}$.

Members of the core microbiota that can influence the community structure through strong biotic interactions with the host or with other microbial species are defined as 'hub microorganisms, ${ }^{53}$. Leotiomycetes have been shown to co-occur with Eurotiomycetes in all the plant organs, and in the root with Geoglossomycetes. Leotiomycetes and Geoglossomycetes have been reported as hub taxa in the $V$. angustifolium root-associated microbiota ${ }^{35}$. These authors suggested an important role of these microorganisms in the wild blueberry soil ecosystem, in particular Leotiomycetes, since this class contains many plant pathogens and mycorrhizal fungi ${ }^{28}$ and therefore might influence plant health and the microbiota associated.

Interestingly, the core fungal community of $V$. myrtillus also included well-established ErMF taxa, such as Pezoloma ericae and Meliniomyces spp., as well as putative ErMF species (Geomyces ${ }^{54}$ ). In the core microbiota were also DSE fungal members of the PAC different from Phialocephala, such 
338

339

340

341

342

343

344

345

as Cadophora, already observed in roots ${ }^{17}$ but never detected in above-ground tissues of Vaccinium spp..

In the genus Oidiodendron, O. maius is the only species known to form ErM and has never been reported in stems or leaves of ericaceous plants so far. In our metabarcoding experiment, a single OTU was identified as O. maius, and although it was not among the core OTUs in V. myrtillus, we detected some reads in two leaf samples. Sparse colonization of the above-ground organs by $O$. maius was also suggested by the results of re-inoculation of Oidiodendron isolates in V. myrtillus under axenic conditions and amplification of fungal DNA from the aerial plant parts. This feature was not unique to O. maius, as fungal DNA from stems and leaves could be amplified for both mycorrhizal and non-mycorrhizal Oidiodendron species. Unfortunately, P. ericae and Meliniomyces spp. were not among the fungi isolated from $V$. myrtillus roots, and direct testing of their ability to colonize the plant systemically in vitro is missing.

These findings suggest that some fungi reported to be exclusively associated with roots, like mycorrhizal and DSE fungi, may colonize other plant organs as well. Interestingly, ErMF and DSE fungi are taxonomically placed within the class Helotiales, which includes many endophytic fungal species $^{28}$. Moreover, a possible life strategy of both ErMF and DSE fungi as endophytes of aboveground plant organs would match the similarities of their genomic features ${ }^{42,43,55}$ with those of other fungal endophytes colonizing aerial plant parts, such as Sarocladium brachiariae in the Sordariomycetes $^{56}$. Common features are the large number of genes involved in cell wall degradation and secondary metabolites biosynthesis, such as polyketide synthases.

Although they can promote plant growth in harsh environments ${ }^{20}$, the mode of action of root DSE fungi is elusive ${ }^{57}$, whereas promotion of host growth and fitness by ErMF has been ascribed to plant-fungus interactions occurring at the symbiotic interface formed around the intracellular fungal coils. If these fungi play any role promoting plant survival in the aerial plant compartments, other so far unknown mechanisms may take place.

In conclusion, we have described by metabarcoding the diversity of fungi associated with the endosphere of below-ground and, for the first time, above-ground organs of V. myrtillus. The results have significantly increased our knowledge of the $V$. myrtillus fungal microbiota and revealed that fungal strains so far considered as strict root symbionts can occupy different niches within the plant, as they were detected in above-ground organs as well.

Several examples of fungi displaying dual life niches have been reported ${ }^{58}$. In particular, ErMF were already known to behave as dual saprotrophs/symbionts, with different root-interacting strategies according to the plant hosts ${ }^{41}$. Here, we show that they may occupy a further ecological niche as 
stem/leaves endophytes. This hypothesis will require further investigations, such as the isolation of ErMF from field-collected V. myrtillus stems/leaves and/or plant inoculation in vitro ${ }^{59}$.

\section{Methods}

\section{Sampling site and description}

The sampling site $\left(45^{\circ} 50^{\prime} 40^{\prime \prime} \mathrm{N}, 7^{\circ} 34^{\prime} 41^{\prime}\right.$ E, $2200 \mathrm{~m}$ a.s.1.) was a subalpine meadow, unused for 10 years, associated with the ICOS network (Integrated Carbon Observation System; station ID: ITTor) and managed by ARPA Valle d'Aosta (Regional Agency for the Environment Protection). In this site dominant taxa were different Vaccinium species ( $V$. myrtillus, $V$. gaultheroides, $V$. vitisidaea), Rhododendron sp., Juniperus sp., Larix decidua. Five clumps of soil with understorey vegetation were collected and stored at $4^{\circ} \mathrm{C}$ overnight. Soil was then washed away and roots of $V$. myrtillus were manually separated from roots of other plant species. The $V$. myrtillus roots were further washed (at least 2 h 30' under running tap water) to remove any soil residues. Separate pools of roots, stems, leaves and flowers of plants from each clump were surface sterilized in $\mathrm{NaClO} 1 \%$ for $1 \mathrm{~min}$ and washed five times with sterile distilled $\mathrm{H}_{2} \mathrm{O}$. We thus collected five pooled samples ( $n=5$, one from each clump) of the four different plant organs. From each pool, at least 4 subsamples of each organ were obtained and immediately stored at $80^{\circ}$. In addition, three root subsamples were not frozen and were used for the isolation of culturable fungi (see below). All the plant experiments were in compliance with relevant institutional, national, and international guidelines and legislation.

\section{DNA extraction, amplification and sequencing.}

The total DNA was extracted (NucleoSpin Plant II, Macherey-Nagel) from at least four subsamples of each organ. The ITS2 region was amplified by a two round PCR: 1) the full ITS region was amplified from the DNA extract with primers ITS1F (5'-CTTGGTCATTTAGAGGAAGTAA-3') and the ITS4 (5'-TCCTCCGCTTATTGATATGC-3'); 2) the ITS2 region was amplified, by a seminested approach, from the product of the first amplification $(1: 10 \mathrm{v} / \mathrm{v}$ dilution $)$ with primers ITS9fngs (5'-GAACGCAGCRAAIIGYGA-3') and ITS4ngs (5'- TCCTCCGCTTATTGATATGC3'), both added to Illumina overhang adapter sequences: forward overhang 5'TCGTCGGCAGCGTCAGATGTGTATAAGAGACAG-[locus specific target primer]-3', reverse overhang: 5'- GTCTCGTGGGCTCGGAGATGTGTATAAGAGACAG-[locus specific target primer]-3'. The obtained PCR products were checked on 1\% agarose gel. The products obtained from different subsamples of the same sample were pooled together before being purified (Wizard SV Gel and PCR CleanUp System, Promega), quantified with Qubit 2.0 (Thermo Fisher Scientific, 
405 Waltham, MA, USA) and sent for Illumina MiSeq 2x300bp sequencing to IGA Technology Services 406 Srl (Udine, Italy).

\section{Bioinformatics}

409 Sequencing adapters and primers were removed and then paired-end reads from each sample were 410 merged with Pear v.0.9. $2^{60}$ using a quality score threshold set at 28 and a minimum length after 411 trimming set at $200 \mathrm{bp}$. The assembled reads were then processed using the Quantitative Insights 412 into Microbial Ecology (Qiime) v.1.9.1 software package ${ }^{61}$. Sequence processing and sample 413 assignment were performed with a minimum sequence length cut-off of $200 \mathrm{bp}$ and a Phred quality 414 score of 28, calculated over a sliding window of $50 \mathrm{bp}$. Chimeric sequences were removed 415 performing a de novo detection using $\mathrm{UCHIME}^{62}$.OTUs were obtained using VSEARCH ${ }^{63}$ at $97 \%$ 416 similarity, and taxonomically assigned using the Full UNITE+INSD dataset for Fungi Version 417 01.12.2017 (UNITE Community 2017). BLAST algorithm ${ }^{64}$ was used as taxonomy assignment 418 method, with $1 \mathrm{e}^{-5}$ e-value as threshold.

419 The statistical and visual analyses on the OTUs have been performed by the Marker Data Profiling 420 tool of MicrobiomeAnalist ${ }^{65}$. OTUs for which at least $10 \%$ of their counts in the different samples 421 contained at least 10 reads have been retained. OTUs with a standard deviation lower than $5 \%$ 422 throughout the experimental conditions were discarded. Data were rarefied to the sample with the 423 lowest sequencing depth. Data have not been scaled a priori.

\section{Isolation of endophytes from roots}

426 Roots were homogenized in sterile water by a sterile glass potter. The homogenized root suspension 427 was centrifuged and washed three times with distilled sterile water; the supernatant was then 428 discarded, and the pellet was suspended in sterile water and plated on MEA medium (2\% malt 429 extract, 1,8\% agar) amended with antibiotics (15 mg/1 streptomycin and $50 \mathrm{mg} / 1$ chloramphenicol).

430 The plates were incubated at $25^{\circ} \mathrm{C}$ and as soon as fungal colonies appeared, they were individually 431 transferred to fresh plates for subsequent identification by morphological and/or molecular analyses. 432 Molecular identification was performed by genomic DNA extraction, followed by PCR 433 amplification of the ITS2 region, Sanger sequencing and Blast search on both NCBI nucleotide 434 library and UNITE database. Some of the isolated strains have been deposited at the Mycotheca 435 Universitatis Taurinensis -MUT- collection of the University of Turin, Italy; see Table S2).

Phylogenetic analysis of fungal isolates in the Helotiales 
438 Blast search of the ITS2 sequences of a few fungal isolates placed them in the Helotiales. A more 439 precise assignment of these isolates in the Helotiales was supported by the phylogenetic analysis 440 performed according to published methods ${ }^{29}$. Briefly, Bayesian analysis and Maximum Likelihood 441 approaches were used for phylogenetic tree construction using MrBayes v. 3.2.6 ${ }^{66}$ and MEGAX ${ }^{67}$, 442 after alignment with ClustalW with default parameters. Reference sequences from public databases 443 are listed in Table S4 ${ }^{29,68}$.

\section{Colonization of V. myrtillus plants in vitro}

446 The Helotiales strains isolated in this work and an O. maius isolate already characterized for its 447 ErM forming abilities (OmMUT1381, deposited at the MUT) were co-cultivated with V. myrtillus 448 seedlings according to a standardized protocol for mycorrhizal synthesis ${ }^{69}$. Root colonization by 449 fungal hyphae of six isolates, three of them expected to be mycorrhizal, was monitored by bright 450 field microscopy after staining with $0.1 \%$ (w/v) cotton blue and destaining overnight with $80 \%$ 451 lactic acid.

452 Fungal colonization of the above-ground organs was checked by PCR, following the same protocols 453 used for DNA extraction and PCR amplification of the fungal ITS2 region from field collected 454 plants (see above). Genomic DNA from OmMUT1381, OmMUT1348 ${ }^{70}$, a basidiomycete and $V$. 455 myrtillus were used as controls in the PCR reaction.

\section{References}

1. Hardoim, P. R. et al. The hidden world within plants: ecological and evolutionary considerations for defining functioning of microbial endophytes. Microbiol. Mol. Biol. Rev. 79, 293-320 (2015).

2. Trivedi, P., Leach, J. E., Tringe, S. G., Sa, T. \& Singh, B. K. Plant-microbiome interactions: from community assembly to plant health. Nat. Rev. Microbiol. 18, 607-621 (2020).

3. Rodriguez, R. J., White Jr, J. F., Arnold, A. E. \& Redman, R. S. Fungal endophytes: diversity and functional roles. New Phytol. 182, 314-330 (2009).

4. Christian, N., Sullivan, C., Visser, N. D. \& Clay, K. Plant host and geographic location drive endophyte community composition in the face of perturbation. Microb. Ecol. 72, 621-632 (2016). 
5. Bamisile, B. S., Dash, C. K., Akutse, K. S., Keppanan, R. \& Wang, L. Fungal endophytes: beyond herbivore management. Front. Microbiol. 9, 544 (2018).

6. Rodriguez, R. J. et al. Stress tolerance in plants via habitat-adapted symbiosis. ISME J. 2, 404416 (2008).

7. Sun, C. et al. Piriformospora indica confers drought tolerance in Chinese cabbage leaves by stimulating antioxidant enzymes, the expression of drought-related genes and the plastidlocalized CAS protein. J. Plant Physiol. 167, 1009-1017 (2010).

8. Clay, K. \& Schardl, C. Evolutionary origins and ecological consequences of endophyte symbiosis with grasses. Am. Nat. 160, S99-S127 (2002).

9. Peterson, R. L. \& Massicotte, H. B. Exploring structural definitions of mycorrhizas, with emphasis on nutrient-exchange interfaces. Can. J. Bot. 82, 1074-1088 (2004).

10. Wilson, D. Endophyte: the evolution of a term, and clarification of its use and definition. Oikos 73, 274 (1995).

11. Qian, X. et al. Leaf and root endospheres harbor lower fungal diversity and less complex fungal co-occurrence patterns than rhizosphere. Front. Microbiol. 10, 1015 (2019).

12. Schwery, O. et al. As old as the mountains: the radiations of the Ericaceae. New Phytol. 207, 355-367 (2015).

13. Meharg, A. A. \& Cairney, J. W. G. Co-evolution of mycorrhizal symbionts and their hosts to metal-contaminated environments. in Advances in Ecological Research vol. 30 69-112 (Elsevier, 1999).

14. Bradley, R., Burt, A. J. \& Read, D. J. Mycorrhizal infection and resistance to heavy metal toxicity in Calluna vulgaris. Nature 292, 335-337 (1981).

15. Hazard, C., Gosling, P., Mitchell, D. T., Doohan, F. M. \& Bending, G. D. Diversity of fungi associated with hair roots of ericaceous plants is affected by land use. FEMS Microbiol. Ecol. 87, 586-600 (2014).

16. Hamim, A. et al. Diversity of fungal assemblages in roots of Ericaceae in two Mediterranean contrasting ecosystems. C. R. Biol. 340, 226-237 (2017). 
17. Koizumi, T. \& Nara, K. Communities of putative ericoid mycorrhizal fungi isolated from alpine dwarf shrubs in japan: effects of host identity and microhabitat. Microbes Environ. 32, 147-153 (2017).

18. Zhang, Y. et al. Diversity of root-associated fungi of Vaccinium mandarinorum along a human disturbance gradient in subtropical forests, China. J. Plant Ecol. 10, 56-66 (2017).

19. Yang, H. et al. Diversity and characteristics of colonization of root-associated fungi of Vaccinium uliginosum. Sci. Rep. 8, 15283 (2018).

20. Newsham, K. K. A meta analysis of plant responses to dark septate root endophytes. New Phytol. 190, 783-793 (2011).

21. Lukešová, T., Kohout, P., Větrovský, T. \& Vohník, M. The potential of dark septate endophytes to form root symbioses with ectomycorrhizal and ericoid mycorrhizal middle European forest plants. PLOS ONE 10, e0124752 (2015).

22. Rimando, A. M., Kalt, W., Magee, J. B., Dewey, J. \& Ballington, J. R. Resveratrol, pterostilbene, and piceatannol in Vaccinium berries. J. Agric. Food Chem. 52, 4713-4719 (2004).

23. Brody, A. K. et al. Genotype specific effects of ericoid mycorrhizae on floral traits and reproduction in Vaccinium corymbosum. Am. J. Bot. 106, 1412-1422 (2019).

24. Tong, X., Shen, X.-Y. \& Hou, C.-L. Antimicrobial activity of fungal endophytes from Vaccinium dunalianum var. urophyllum. Sains Malays. 47, 1685-1692 (2018).

25. Zhang, Y.-H. Z. Phylogenetic relationships of some members in the genus Hymenoscyphus (Ascomycetes, Helotiales). Nova Hedwig. 78, 475-484 (2004).

26. Pearson, V. \& Read, D. J. The biology of mycorrhiza in the Ericaceae. II. The transport of carbon and phosphorus by the endophyte and the mycorrhiza. New Phytol. 72, 1325-1331 (1973).

27. Vrålstad, T., Fossheim, T. \& Schumacher, T. Piceirhiza bicolorata - the ectomycorrhizal expression of the Hymenoscyphus ericae aggregate? New Phytol. 145, 549-563 (2000).

28. Leopold, D. R. Ericoid fungal diversity: challenges and opportunities for mycorrhizal research. Fungal Ecol. 24, 114-123 (2016). 
29. Fehrer, J., Réblová, M., Bambasová, V. \& Vohník, M. The root-symbiotic Rhizoscyphus ericae aggregate and Hyaloscypha (Leotiomycetes) are congeneric: phylogenetic and experimental evidence. Stud. Mycol. 92, 195-225 (2019).

30. Lacourt, I. et al. Nuclear ribosomal sequence analysis of Oidiodendron 
39. Li, Z.-J., Shen, X.-Y. \& Hou, C.-L. Fungal endophytes of South China blueberry (Vaccinium dunalianum var. urophyllum ). Lett. Appl. Microbiol. 63, 482-487 (2016).

40. Koudelková, B., Jarošová, R. \& Koukol, O. Are endophytic fungi from Rhododendron tomentosum preadapted for its essential oil? Biochem. Syst. Ecol. 75, 21-26 (2017).

41. Vohník, M. et al. The cultivable endophytic community of Norway spruce ectomycorrhizas from microhabitats lacking ericaceous hosts is dominated by ericoid mycorrhizal Meliniomyces variabilis. Fungal Ecol. 6, 281-292 (2013).

42. Martino, E. et al. Comparative genomics and transcriptomics depict ericoid mycorrhizal fungi as versatile saprotrophs and plant mutualists. New Phytol. 217, 1213-1229 (2018).

43. Schlegel, M. et al. Globally distributed root endophyte Phialocephala subalpina links pathogenic and saprophytic lifestyles. BMC Genomics 17, 1015 (2016).

44. Segata, N. et al. Metagenomic biomarker discovery and explanation. Genome Biol. 12, R60 (2011).

45. Fang, K. et al. Tissue-specific and geographical variation in endophytic fungi of Ageratina adenophora and fungal associations with the environment. Front. Microbiol. 10, 2919 (2019).

46. Bálint, M. et al. Host genotype shapes the foliar fungal microbiome of balsam poplar (Populus balsamifera). PLoS ONE 8, e53987 (2013).

47. Toju, H., Kurokawa, H. \& Kenta, T. Factors influencing leaf- and root-associated communities of bacteria and fungi across 33 plant orders in a grassland. Front. Microbiol. 10, 241 (2019).

48. de Vries, R. P., de Lange, E. S., Wösten, H. A. B. \& Stalpers, J. A. Control and possible applications of a novel carrot-spoilage basidiomycete, Fibulorhizoctonia psychrophila. Antonie Van Leeuwenhoek 93, 407-413 (2008).

49. Larsen, M. J., Jurgensen, M. F. \& Harvey, A. E. Athelia epiphylla associated with colonization of subalpine fir foliage under psychrophilic conditions. Mycologia 73, 1195-1202 (1981).

50. Yurchenko, E. O. \& Golubkov, V. V. The morphology, biology, and geography of a necrotrophic basidiomycete Athelia arachnoidea in Belarus. Mycol. Prog. 2, 275-284 (2003).

51. Nam, M. H., Park, M. S., Kim, H. S., Kim, T. I. \& Kim, H. G. Cladosporium cladosporioides and C. tenuissimum cause blossom blight in strawberry in korea. Mycobiology 43, 354-359 (2015). 
52. Grünig, C. R., Queloz, V., Sieber, T. N. \& Holdenrieder, O. Dark septate endophytes (DSE) of the Phialocephala fortinii s.l. - Acephala applanata species complex in tree roots: classification, population biology, and ecology. Botany 86, 1355-1369 (2008).

53. Hamonts, K. et al. Field study reveals core plant microbiota and relative importance of their drivers. Environ. Microbiol. 20, 124-140 (2018).

54. Vohník, M., Fendrych, M., Albrechtová, J. \& Vosátka, M. Intracellular colonization of Rhododendron and Vaccinium roots by Cenococcum geophilum, Geomyces pannorum and Meliniomyces variabilis. Folia Microbiol. (Praha) 52, 407-414 (2007).

55. Knapp, D. G. et al. Comparative genomics provides insights into the lifestyle and reveals functional heterogeneity of dark septate endophytic fungi. Sci. Rep. 8, 6321 (2018).

56. Yang, Y. et al. Genomic characteristics and comparative genomics analysis of the endophytic fungus Sarocladium brachiariae. BMC Genomics 20, 782 (2019).

57. Mandyam, K. \& Jumpponen, A. Seeking the elusive function of the root-colonising dark septate endophytic fungi. Stud. Mycol. 53, 173-189 (2005).

58. Selosse, M.-A., Schneider-Maunoury, L. \& Martos, F. Time to re-think fungal ecology? Fungal ecological niches are often prejudged. New Phytol. 217, 968-972 (2018).

59. Vohník, M. Ericoid mycorrhizal symbiosis: theoretical background and methods for its comprehensive investigation. Mycorrhiza 30, 671-695 (2020).

60. Zhang, J., Kobert, K., Flouri, T. \& Stamatakis, A. PEAR: a fast and accurate Illumina PairedEnd reAd mergeR. Bioinforma. Oxf. Engl. 30, 614-620 (2014).

61. Caporaso, J. G. et al. QIIME allows analysis of high-throughput community sequencing data. Nat. Methods 7, 335-336 (2010).

62. Edgar, R. C. Search and clustering orders of magnitude faster than BLAST. Bioinforma. Oxf. Engl. 26, 2460-2461 (2010).

63. Rognes, T., Flouri, T., Nichols, B., Quince, C. \& Mahé, F. VSEARCH: a versatile open source tool for metagenomics. PeerJ 4, e2584 (2016).

64. Altschul, S. F., Gish, W., Miller, W., Myers, E. W. \& Lipman, D. J. Basic local alignment search tool. J. Mol. Biol. 215, 403-410 (1990). 
65. Chong, J., Liu, P., Zhou, G. \& Xia, J. Using MicrobiomeAnalyst for comprehensive statistical, functional, and meta-analysis of microbiome data. Nat. Protoc. 15, 799-821 (2020).

66. Ronquist, F. \& Huelsenbeck, J. P. MrBayes 3: Bayesian phylogenetic inference under mixed models. Bioinforma. Oxf. Engl. 19, 1572-1574 (2003).

67. Kumar, S., Stecher, G., Li, M., Knyaz, C. \& Tamura, K. MEGA X: Molecular Evolutionary Genetics Analysis across computing pPlatforms. Mol. Biol. Evol. 35, 1547-1549 (2018).

68. Wei, X., Chen, J., Zhang, C. \& Pan, D. A New Oidiodendron maius strain isolated from Rhododendron fortunei and its effects on nitrogen uptake and plant growth. Front. Microbiol. 7, (2016).

69. Casarrubia, S. et al. The Hydrophobin-Like OmSSP1 may be an effector in the ericoid mycorrhizal symbiosis. Front. Plant Sci. 9, 546 (2018).

70. Martino, E., Perotto, S., Parsons, R. \& Gadd, G. M. Solubilization of insoluble inorganic zinc compounds by ericoid mycorrhizal fungi derived from heavy metal polluted sites. Soil Biol. Biochem. 35, 133-141 (2003).

\section{Acknowledgments}

460 This research was partly supported by local funding (ex-60\%) from the University of Turin.

461 The authors thank Dr. L. Oddi for her support in sample collection and for the botanical description 462 of the sampling site. The Authors are also particularly thankful to Chiara Maggi and Chiara 463 Scapaticci for their help in performing some of the experiments during their undergraduate 464 internship.

\section{Author contributions}

467 The project was conceived by SD, SP and EM. The in field sampling and sample treatment in laboratory was done by SD and EM. SD carried out fungal isolation, DNA extraction, amplification and quality check before sequencing, as well as the molecular identification of the isolates. SV performed the morphological identification of the isolates, the bioinformatic analysis of the raw sequencing data and helped in the statistical analysis. SD performed all the analysis based on the

472 Microbiome Analist web-tool. The manuscript was largely written by SD, with thorough revision by 473 all the authors. 


\section{Data availability statement}

476 The raw sequences from the metabarcoding experiment have been deposited with the BioProject ID 477 PRJNA769432 (https://www.ncbi.nlm.nih.gov/bioproject/?term=PRJNA769432). The data-sets 478 generated and analyzed during the current study are included in this published article (and its 479 Supplementary Information files).

\section{Competing interests}

482 The authors declare no competing interests.

\section{Figure legends}

Figure 1. Beta-diversity of the fungal communities associated with the different plant organs. The beta-diversity among the different organs was estimated by a NMDS analysis based on Bray-Curtis dissimilarities, with the following parameters: taxonomic level: feature, statistical method: PERMANOVA, experimental factor: organ. Fi=Flowers, Fo=Leaves, Fu=Stems, R=Roots. C1-C5: samples.

Figure 2. Fungal classes associated with different plant organs. (a) relative abundance of classes,

(b) classes with statistically different abundance among the organs (Pairwise Wilcoxon rank sum test/Kruskal-Wallis with BH-adjusted $\mathrm{p}$-val<0.05).

Figure 3. Heat tree matrix depicting the different taxa abundance among the plant organs, for all orders in the dataset. The size of the nodes in the gray cladogram (right) represents the number of OTUs identified at that taxonomic level. The small cladograms show the pairwise comparisons among the organs: a yellow node indicates a higher abundance of the taxon in the organ indicated in yellow, than in the organ indicated in green. A green node indicates the opposite. Taxa identified as differently represented, statistically supported by the Wilcoxon test $(\mathrm{p}<0.05)$, are tagged with a white asterisk.

Figure 4. Hub taxa in each organ defined by Linear discriminant analysis Effect Size (LefSe). a) classes, b) orders and c) genera that best characterize each organ (LDA score $>3$ and Kruskal-Wallis $\mathrm{p}$-val<0.05) are ranked in decreasing order by their LDA scores ( $\mathrm{x}$ axis). The mini-heatmap to the right of the plot indicates whether the taxa are more (red) or less (blue) represented in each organ. 
509 Figure 5. Heatmap of the core OTUs genera. A double clustering based on average linkage 510 algorithm and Pearson correlation, that clusters together features or samples with similar behavior, 511 has been performed.

512

513 Figure 6. V. myrtillus roots colonized by O. maius (a-b-c), O. tenuissimum (d), and Cadophora 514 luteo-olivacea (e-f) strains. The strains have been isolated in this work and correspond to isolates a) 515 M2-5; b) M30; c) M44, d) M42, e) M17, f) M29 (see Supplementary Table S2). The white stars 516 indicate intracellular hyphal coils. 


\section{Figures}

\section{Figure 1}

Beta-diversity of the fungal communities associated with the different plant organs. The beta-diversity among the different organs was estimated by a NMDS analysis based on Bray-Curtis dissimilarities, with the following parameters: taxonomic level: feature, statistical method: PERMANOVA, experimental factor: organ. Fi=Flowers, Fo=Leaves, Fu=Stems, R=Roots. C1-C5: samples.

\section{Figure 2}

Fungal classes associated with different plant organs. (a) relative abundance of classes, (b) classes with statistically different abundance among the organs (Pairwise Wilcoxon rank sum test/Kruskal-Wallis with $\mathrm{BH}$-adjusted $\mathrm{p}$-val<0.05).

\section{Figure 3}

Heat tree matrix depicting the different taxa abundance among the plant organs, for all orders in the dataset. The size of the nodes in the gray cladogram (right) represents the number of OTUs identified at that taxonomic level. The small cladograms show the pairwise comparisons among the organs: a yellow node indicates a higher abundance of the taxon in the organ indicated in yellow, than in the organ indicated in green. A green node indicates the opposite. Taxa identified as differently represented, statistically supported by the Wilcoxon test $(p<0.05)$, are tagged with a white asterisk.

\section{Figure 4}

Hub taxa in each organ defined by Linear discriminant analysis Effect Size (LefSe). a) classes, b) orders and c) genera that best characterize each organ (LDA score $>3$ and Kruskal-Wallis $p$-val $<0.05$ ) are ranked in decreasing order by their LDA scores ( $x$ axis). The mini-heatmap to the right of the plot indicates whether the taxa are more (red) or less (blue) represented in each organ. (d) Principal Component Analysis showing the distances between the samples.

\section{Figure 5}


Heatmap of the core OTUs genera. A double clustering based on average linkage algorithm and Pearson correlation, that clusters together features or samples with similar behavior, has been performed.
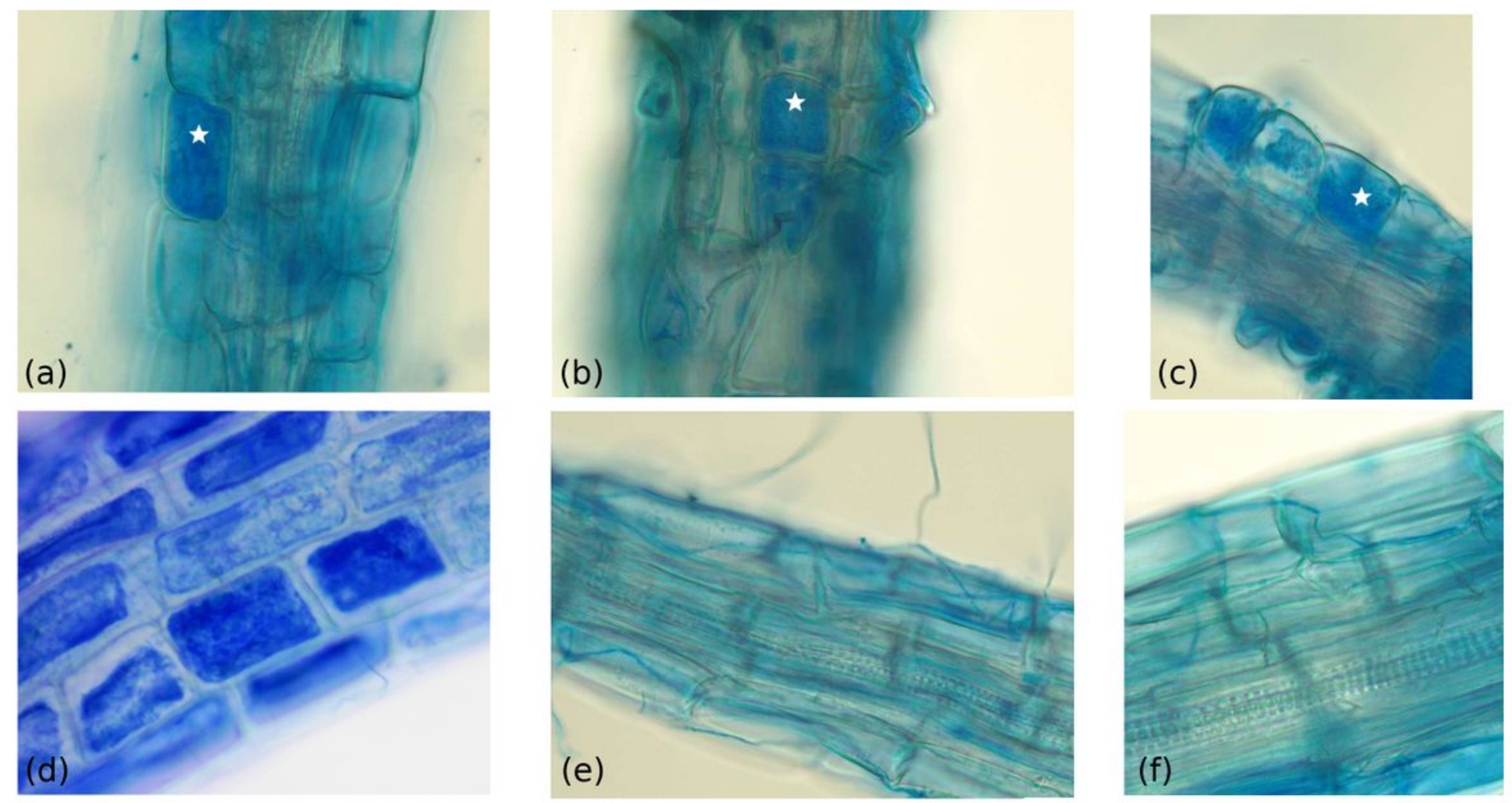

\section{Figure 6}

V. myrtillus roots colonized by 0 . maius (a-b-c), 0 . tenuissimum (d), and Cadophora luteo-olivacea (e-f) strains. The strains have been isolated in this work and correspond to isolates a) M2-5; b) M30; c) M44, d) M42, e) M17, f) M29 (see Supplementary Table S2). The white stars indicate intracellular hyphal coils.

\section{Supplementary Files}

This is a list of supplementary files associated with this preprint. Click to download.

- DaghinoetalSupplementaryfigurescaptions.pdf

- TableS1.xIsx 